Original Paper http://ajol.info/index.php/ijbcs http://indexmedicus.afro.who.int

\title{
Evaluation de la substitution du tourteau de Glycine max (L.) Merr. (Soja) par le tourteau de Tetracarpidium conophorum (Müll. Arg.) Hutch. \& Dalz sur les performances des poules pondeuses
}

\author{
Vincy Midrak NTSOUMOU ${ }^{1}$, Prudence Pitchou ADZONA ${ }^{2}$, Jean Bamard BATI ${ }^{3}$, \\ Tédia KENGUE ${ }^{2}$, Balthazar Bienvenu MABANZA-MBANZA ${ }^{3}$, \\ Alain Juste SABOUKOULOU ${ }^{1}$, Arnaud Fiacre NDINGA ${ }^{3}$, Joseph Rusty GUEMBO ${ }^{1}$ et \\ Henri BANGA-MBOKO ${ }^{2 *}$
}

\author{
${ }^{1}$ Faculté des Sciences et Techniques, Université Marien NGOUABI P. B 69 Brazzaville-Congo. \\ ${ }^{2}$ Ecole Nationale d'Agronomie et de Foresterie, Université Marien NGOUABI P. B 69 Brazzaville-Congo. \\ ${ }^{3}$ Institut national de Recherche Agronomique (IRA), avenue des premiers jeux africains, face stade Alphonse \\ Massamba-Débat, Brazzaville, Congo. \\ *Auteur correspondant ; E-mail:hbangamboko@ gmail.com ; Tel.: (+242) 0668514761055778025
}

\begin{tabular}{lll}
\hline Received: 09-07-2021 & Accepted: 26-11-2021 & Published: 31-12-2021 \\
\hline
\end{tabular}

\section{RESUME}

Tetracarpidium conophorum est une liane sauvage qui produit l'huile à des fins cosmétiques. Malheureusement, son tourteau bien que riche en protéines, n'a pas encore été testé dans l'alimentation animale, malgré les couts élevés, l'importation et l'irrégularité du tourteau de soja (la source de protéines la plus utilisée). L'objectif de ce travail était d'évaluer l'effet d'une substitution du tourteau de soja par le tourteau de $T$. conophorum sur les performances zootechniques et économiques des poules pondeuses. Un échantillon de 63 pondeuses de la souche ISA Brown, âgées de 35 semaines a été réparti à poids sensiblement égal en trois lots de 21 sujets chacun ( $1480 \mathrm{~g}$ pour le lot témoin ; $1500 \mathrm{~g}$ pour le lot traité 1 et $1490 \mathrm{~g}$ pour le lot traité 2 ). Chaque lot était subdivisé en trois répétitions de sept poules chacune ; le lot témoin (T0) a reçu un aliment ne contenant pas du $T$ conophorum ; le lot traité 1 (T6) un aliment contenant équitablement $6 \%$ (tourteau de T. conophorum et tourteau de soja), tandis que le lot 2 (T12) a reçu un aliment contenant $12 \%$ du tourteau T. conophorum et sans le tourteau de Glycine max. Les résultats ont montré que la substitution a amélioré significativement le nombre d'œufs pondus par semaine par poule $(5,46$ contre 5,64 et 6,14$)$, la masse d'œufs par poule par semaine (314 g contre $319 \mathrm{~g}$ et $350 \mathrm{~g})$, le taux de ponte $(80,10 \%$ contre $90,22 \%$ et $82,65 \%)$, l'indice de consommation $(2,80$ contre 2,31et 2,49), le prix de revient d'un kg d'aliment (332 contre 317 et 295 FCFA), ainsi que le coût de production de l'œuf ( 89,50 contre 77,49 et 77, 68 FCFA). Cette étude suggère que le tourteau de T. conophorum à $12 \%$ peut remplacer totalement le tourteau soja sans affecter les performances des poules pondeuses et ouvre des perspectives intéressantes en aviculture fermière en zone tropicale.

(C) 2021 International Formulae Group. All rights reserved.

Mots clés : Noix africaine, ISA Brown, Alimentation, œufs, coût de production, République du Congo. 


\title{
Evaluation of the substitution of the Glycine max (L.) Merr. (Soya) bean oil cake by oil cake of Tetracarpidium conophorum (Müll. Arg.) Hutch. \& Dalz on the performances of laying hens
}

\begin{abstract}
Tetracarpidium conophorum, a wild liana that fruits produces a cosmetic oil, however, its oil cake is not include in the animal feedings, despite, the high cost of soya oil cake. Therefore, the objective of this work was to evaluate the substitution effect of the soya bean oil cake by oil cake of T. conophorum on laying hens. A sample of 63 laying aged 35 week old had been separated in to three groups. Each group was equally randomized and separated in three replicates of 7 laying hens each. Three diets were used: T0 (T. conophorum 0\%; with $12 \%$ oilcake soya)? T6 (6\% T. conophorum and $6 \%$ of oil cake soya) and finally T12 (12\% of the oil cake $T$. conophorum and $0 \%$ of the soya bean oil cake). Data showed that substitution by 6 or $12 \%$ significantly improved the number of eggs laid per week by hen on average (5.46 versus 5.64 and 6.14), the egg mass per hen (314 g versus $319 \mathrm{~g}$ and $350 \mathrm{~g}$ ), the rate of laying $(80.10 \%$ versus $90.22 \%$ and $82.65 \%)$, the index of consumption $(2.80$ versus 2.49 and 2.31), the cost of one $\mathrm{kg}$ of feed (332 versus 317 and 295) FCFA, as well as the production costs of the egg (89.50 versus 77.49 and 77.68) FCFA respectively T0 versusT1 and T2. The study indicated that $T$. conophorum oil cake at $12 \%$ may completely replace the oil cake soya without affecting the performances of layers. The incorporation of oil cake $T$. conophorum in laying hens may increase production and profitability in small scale poultry farming in tropical areas.
\end{abstract}

(C) 2021 International Formulae Group. All rights reserved.

Keywords: African nut, ISA Brown, feed, eggs, production costs, Congo.

\section{INTRODUCTION}

Les tourteaux constituent des résidus solides obtenus à partir de l'extraction d'huiles des graines oléo-protéagineuses. Ils contiennent des protéines de haute valeur nutritionnelle et entrent dans les proportions de $16-20 \%$ dans les formulations alimentaires. Le tourteau de soja est à cet effet le plus utilisé dans les rations de volailles et aussi le plus onéreux (Livet et al., 2015 ; Roinsard et al., 2016). Actuellement le prix d'un kg du soja au Congo Brazzaville varie entre 550 et 800 FCFA (Mantsanga et al., 2016). Ce qui entraine de répercussions sur les coûts de production d'un kg d'aliment, du poulet de chair et de l'œuf.

Des solutions alternatives sont envisagées pour le remplacement partiel ou total des intrants alimentaires classiques, par d'autres sources disponibles et moins chers (Brou et al., 2012 ; Guedou et al., 2018). En effet, les ressources alimentaires non conventionnelles peu ou pas valorisées en alimentation de volaille, peuvent constituer des ingrédients alternatifs à moindre coût
(OFIVAL, 2005 ; FAO, 2009a ; Dongmo et al., 2012 ; Houndonougbo et al., 2012 ; Hien et al., 2017).

Au Congo, le manque d'huileries conduit à la rareté des tourteaux. A cet effet, il parait nécessaire de valoriser la production locale ou artisanale des tourteaux non conventionnels.

Plusieurs expériences ont été réalisées sur la valorisation de certains tourteaux locaux non conventionnels comme la courge, le coprah, le sésame et le tourteau de la pulpe de Dacryodes edulis (Adzona, 2019; Adzona, et al., 2019) et le tourteau de Tetracarpidium conophorum (Mezajougkenfack, 2010 ; Sianard, 2010; Malanda Londé, 2016; Uchechukwu-Nkeirukayvonne, 2017 ; Ezugwu et al., 2021 ; Ossoko et al., 2021).

T. conophorum, est une liane sauvage de la famille des Euphorbiacées (Bernadin et al., 2012), oléagineuse, mesurant $30 \mathrm{~m}$ de long (Ayoola et al., 2011). Cette plante regorge les vertus pharmaceutiques, cosmétiques (Oriakhiet Uadia, 2020 ; Ojokoh et al., 2020) ; 
son huile peu connue dans la cuisine est extraite en petite quantité. Son tourteau est richement nutritif avec les teneurs en protéines brutes variant entre $33 \%$ et $47 \%$ suivant la technologie appliquée (Mezajougkenfack, 2010 ; Sianard, 2010 ; Uchechukwu-Nkeirukayvonne, 2017 ; Ossoko et al., 2021).

Le tourteau de soja a fait l'objet d'essais de substitution dans l'alimentation des volailles tant sur la production de la chair que des œufs notamment par les tourteaux de colza, de tournesol, pois, protéine de pomme de terre (Marc et Peter, 2021) chez les poulets de chair et par le tourteau de safou chez les poules pondeuses (Saboukoulou, 2020). La littérature révèle que jusqu'à ce jour, aucune étude de substitution de tourteau de soja par le tourteau de Tetracarpidium conophorum n'a été réalisée.

Il est donc apparu opportun d'initier la réponse des volailles sur des rations contenant du tourteau de Tetracarpiduim conophorum, en substitution avec le tourteau de soja.

C'est pour combler cette lacune que ce travail repose sur l'hypothèse selon laquelle le tourteau de T. conophorum peut substituer le tourteau de soja sans affecter les performances de ponte.

L'objectif de ce travail était d'évaluer l'effet de la substitution du tourteau de soja par le tourteau de Tetracarpidium conophorum sur les performances zootechniques et économiques des poules pondeuses.

\section{MATERIEL ET METHODES}

\section{Zone d'étude}

Cette étude a été réalisée dans deux localités du Congo illustrées par la Figure 1:

Lékana, pour la collecte des noix : les noix provenaient des lianes à l'état sauvage dans le Département des Plateaux, plus précisément dans le District de Lékana, situé à près de 450 kilomètres de la capitale (Brazzaville). Ce district est situé à 790 mètres d'altitude par rapport au niveau de la mer; avec une Latitude de $2019^{\prime}$ 31" Sud et $14^{\circ} 36^{\prime} 00^{\prime}$ "Est de la République du Congo (Anonyme, 2019).

Brazzaville, pour les expérimentations à la ferme expérimentale de
l'Institut national de Recherche Agronomique (IRA), situé dans l'arrondissement 2 Bacongo. Le climat de Brazzaville est tropical humide de type bas-congolais, les températures moyennes annuelles avoisinent $25^{\circ} \mathrm{C}$ avec des faibles écarts thermiques n'excédant pas $5^{\circ} \mathrm{C}$. La température maximale ne dépasse pas $35^{\circ} \mathrm{C}$ et la température minimale reste supérieure à $20^{\circ} \mathrm{C}$ (Defoundoux et al., 2006). Il est caractérisé par deux saisons : une saison de pluies d'octobre à mai avec un fléchissement en janvier et une saison sèche de juin à septembre. Brazzaville est située à près de 301 $\mathrm{m}$ d'altitude par rapport au niveau de la mer ; avec une latitude de $4^{\circ} 15^{\prime} 58^{\prime \prime}$ au Sud et la longitude : $15^{\circ} 16^{\prime} 59^{\prime}$ ' à l'Est de la République du Congo (Anonyme, 2021).

\section{Matériel végétal}

Le matériel végétal ayant fait l'objet de la présente étude était constitué de tourteau de T. conophorum obtenu après extraction d'huile à partir des noix selon la méthode décrite par Mezajoug Kenfack (2010) et Sianard (2010) (Figure 2).

Brièvement, sur $14 \mathrm{~kg}$ de noix brutes, 1 $\mathrm{kg}$ de graines avariées a été trié ; $13 \mathrm{~kg}$ ont fait l'objet d'un traitement thermique durant $3 \mathrm{~h}$. Une quantité de $10,75 \mathrm{~kg}$ d'amandes a été obtenue après décortication; $10 \mathrm{~kg}$ de broyat ont été produits suite au séchage. Enfin, après extraction de $2,25 \mathrm{~kg}$ d'huile par presse mécanique du broyat, $7,75 \mathrm{~kg}$ de tourteaux ont été produits soit un rendement de $77,5 \%$ de tourteau.

\section{Matériel animal et conditions expérimentales \\ Phase pré-expérimentale}

Les poussins d'un jour ont été élevés au sol sur la litière et nourris avec un aliment démarrage jusqu'à 8 semaines, ensuite transférés dans le local de poulettes.

Les poulettes ont été nourries pendant la phase pré-ponte (de la 9ème jusqu'à la $19^{\text {ième }}$ semaine), par un aliment contenant 2856,56 $\mathrm{Kcal} / \mathrm{kg}$ d'énergie et $15,55 \%$ de protéines.

\section{Phase expérimentale}

Les poulettes ont été transférées par la suite dans le local de ponte à l'âge de 20 
semaines et élevées au sol sur la litière constituée du copeau de menuiserie dans les mêmes conditions de température et d'humidité (Sciences et Techniques avicoles, 2004).

Les prises de température et d'humidité ont été effectuées ; les mesures de prophylaxie sanitaire et médicale ont été mises en application suivant un calendrier bien défini pour prévenir d'éventuelles pathologies telles que la coccidiose aviaire, la grippe aviaire, la maladie de gumboro.

\section{Dispositif expérimental}

Un échantillon de 63 pondeuses de la souche ISA Brown âgées de 35 semaines a servi de l'expérimentation Les poules pondeuses ont été réparties de manière aléatoire en trois lots comportant 21 sujets par compartiment et en 3 répétitions de 7 pondeuses chacune comme l'indique le Tableau 1 du dispositif expérimental.

\section{Rations alimentaires}

Les différentes rations ayant fait l'objet de cette étude ont été formulées à base des ingrédients locaux disponibles d'après la méthode développée par Brah et al. (2019).

Le Tableau 2 présente les différentes rations alimentaires utilisées.

\section{Paramètres zootechniques étudiés Contrôle de la température ambiante et d'humidité}

Durant la phase expérimentale, la température et l'humidité ont été mesurées quotidiennement en deux prises (matin et soir) à l'aide d'un thermo-hygromètre placé dans chaque local. Les données sur la prise de température et d'humidité sont consignées dans le Tableau 3.

\section{Mortalité}

Par observation visuelle des sujets, les pondeuses ont été contrôlées chaque jour, tout au long de l'expérimentation.

\section{Consommation volontaire d'aliment}

La consommation volontaire d'aliment (CVA) a été calculée par soustraction des refus (r) sur les quantités distribuées (D).

$$
\text { CVA }=\text { D-r }
$$

\section{Consommation volontaire d'eau}

Tout comme l'aliment, la consommation volontaire d'eau (CVE) a été calculée par soustraction des refus (r) sur les quantités distribuées (D).

$$
\mathrm{CVE}=\mathrm{D}-\mathrm{r}
$$

\section{Indice de consommation}

L'indice de consommation (IC) a été déterminé suivant la formule ci-après.

$$
\begin{aligned}
& \mathrm{IC}=\frac{\text { Quantité } \mathrm{d}^{\prime} \text { aliment consommé }}{\text { Masse } \mathrm{d}^{\prime} \text { oeufs }} \\
& \text { Production des aufs }
\end{aligned}
$$

\section{Production des oufs}

Les variables de production notamment la masse d'œufs et le taux de ponte ont été calculés à base des formules suivantes :

Masse d'œufs = Nombre d'œufs pondus $\mathrm{x}$ poids moyen de l'œuf

Taux de ponte :

$$
\mathrm{TP}=\frac{\text { Nombre d'œufs pondus par jour }}{\text { Nombre de poules présentes }} \times 100
$$

\section{Variables économiques calculées \\ Coût de production de l'aliment}

Le prix de revient d'un kilogramme d'aliment a été déterminé sur la base de : prix d'achat des ingrédients pour chaque régime alimentaire à partir du coût d'un mélange de $100 \mathrm{~kg}$ d'aliment présenté au tableau 6 .

\section{Coût de production de l'ouf}

Durant l'expérimentation, la quantité moyenne d'aliment consommé a été calculée. Sur la base de l'indice de consommation, cumulé aux autres charges d'exploitation, le coût de production de l'œuf a été déterminé comme suit :

La déduction de l'indice de conversion (Ic) de l'aliment en œuf à partir de l'indice de consommation (IC) de chaque lot: $\mathrm{Ic}=\mathrm{IC} / \mathrm{kg}$ d'œufs $(\mathrm{N})$ avec $\mathrm{N}$ : nombre d'œufs pour un kilogramme.

\section{Analyse statistique}

Les données obtenues au cours de cette expérience ont été saisies dans le logiciel Excel, puis exportées et analysées avec le logiciel SAS (Statistical Analysis System) version 2013. Le type de tourteau (soja et $T$. conophorum) a été le seul facteur pour le modèle d'analyse de variance. Le test $\mathrm{F}$ de Fisher a été utilisé pour déterminer la significativité de l'effet du facteur traitement et le $t$ de student est intervenu pour comparer les moyennes deux à deux ( $\mathrm{T} 0 \mathrm{vs} \mathrm{T} 1$ et $\mathrm{T} 0$ vs T2). 

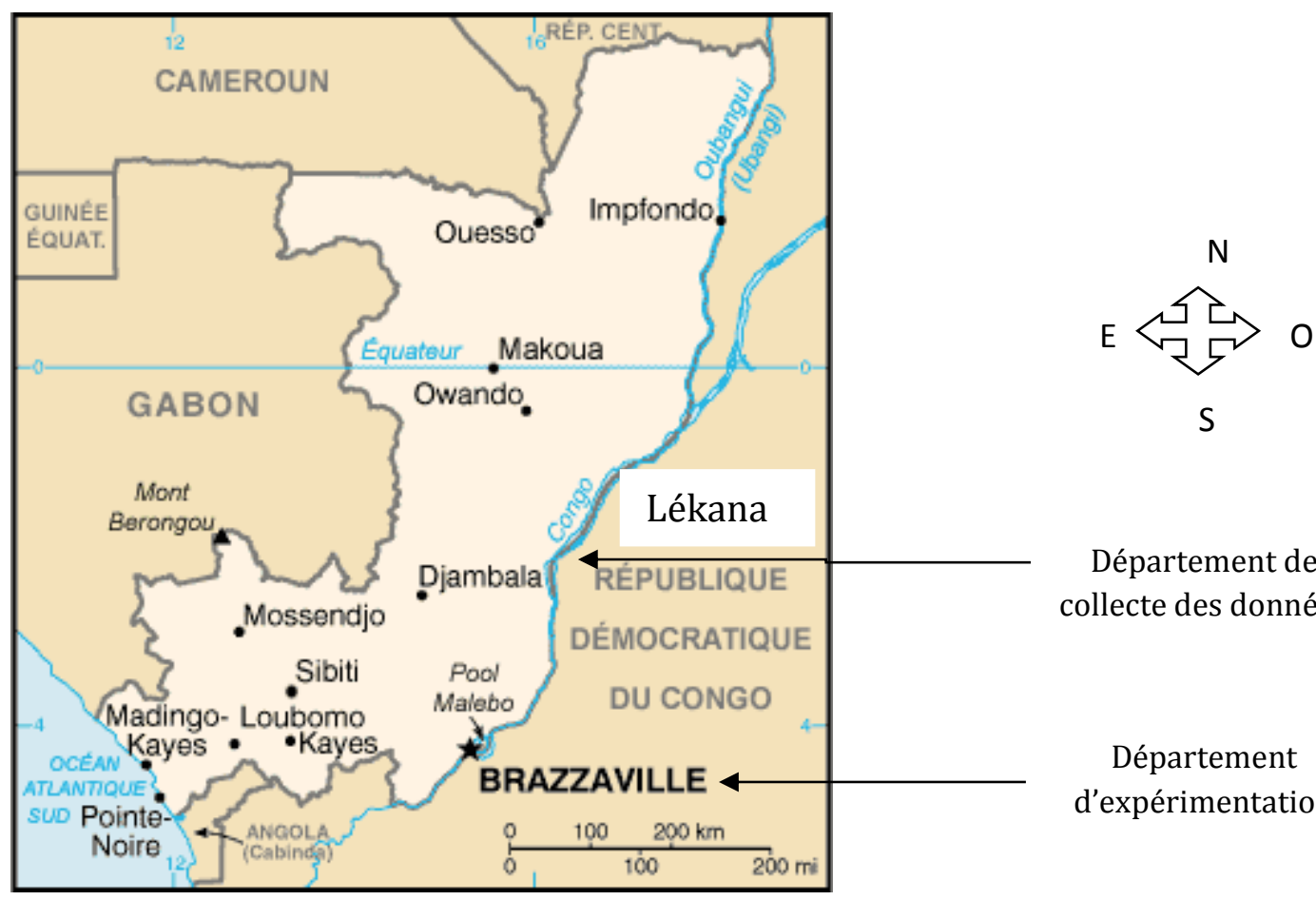

Figure 1 : Zones d'étude.

Source: Anonyme (2011).

Tableau 1: Dispositif expérimental.

\begin{tabular}{lccc}
\hline Nombre de sujets N= 21 & Témoin T0 ; & Traite T1 ; & Traite T2 \\
\hline Taux d'incorporation du soja & $12 \%$ & $6 \%$ & $0 \%$ \\
Taux d'incorporation du TTC & $0 \%$ & $6 \%$ & $12 \%$ \\
Taux de substitution du soja & $0 \%$ & $50 \%$ & $100 \%$ \\
\hline
\end{tabular}

Tableau 2 : Composition et analyse chimique de chaque ration alimentaire.

\begin{tabular}{|c|c|c|c|}
\hline \multirow{2}{*}{$\begin{array}{l}\text { Lots } \\
\text { Ingrédients }\end{array}$} & \multicolumn{3}{|c|}{ Taux d'incorporation (\%) } \\
\hline & T0 & T1 & T2 \\
\hline Maïs grain jaune & 20 & 20 & 20 \\
\hline Farine de graines de mil & 11 & 10 & 11 \\
\hline Farine grossière de manioc & 14 & 13 & 14 \\
\hline Son de blé & 11,5 & 11,5 & 11,5 \\
\hline Huile de palme & 2 & 2 & 2 \\
\hline Farine de poisson & 10 & 10 & 10 \\
\hline Farine de graines de Niébé & 12 & 14 & 12 \\
\hline Tourteau de $T$. conophorum & 0 & 6 & 12 \\
\hline Tourteau de soja & 12 & 6 & 0 \\
\hline Calcaire & 7 & 7 & 7 \\
\hline Sel de cuisine & 0,4 & 0,4 & 0,4 \\
\hline Complexe minéral, aminé vitaminé ${ }^{1}$ & 0,1 & 0,1 & 0,1 \\
\hline Total & 100 & 100 & 100 \\
\hline
\end{tabular}




\begin{tabular}{lccc}
\hline \multicolumn{4}{c}{ Valeur nutrive calculée des régimes alimentaires } \\
\hline Lots & T0 & T1 & T2 \\
\hline Energie Métabolisable (Kcal/kg) & 2802 & 2734 & 2762 \\
Matières Azotées Totales (\%) & 18 & 18 & 18 \\
Lysine (\%) & 1,64 & 1,49 & 1,40 \\
Méthionine (\%) & 0,41 & 0,45 & 0,49 \\
Cellulose brute (\%) & 4,5 & 4,69 & 4,97 \\
Calcium (\%) & 3,5 & 3,53 & 3,55 \\
Phosphore (\%) & 0,62 & 0,71 & 0,80 \\
Ratio EM/MAT & 150 & 151 & 152 \\
\hline
\end{tabular}

${ }^{1}$ Le régime contient d'une part, des vitamines : A (10MUI), D3 (3UI), E (2500 mg), K3 (4000 mg), B1 (5000 mg), B2 ( 500 $\mathrm{mg}), \mathrm{B} 6(2500 \mathrm{mg}), \mathrm{B} 12(5 \mathrm{mg}), \mathrm{C}(10000 \mathrm{mg})$, PP (2000mg), pantothénate de calcium (5000 mg), biotine (5 mg), acide folique $(250 \mathrm{mg})$; d'autre part, des oligoéléments : fer, cuivre, zinc, manganèse, cobalt, magnésium, iode de sodium (70 mg), chlorure de potassium $(15 \mathrm{mg})$.

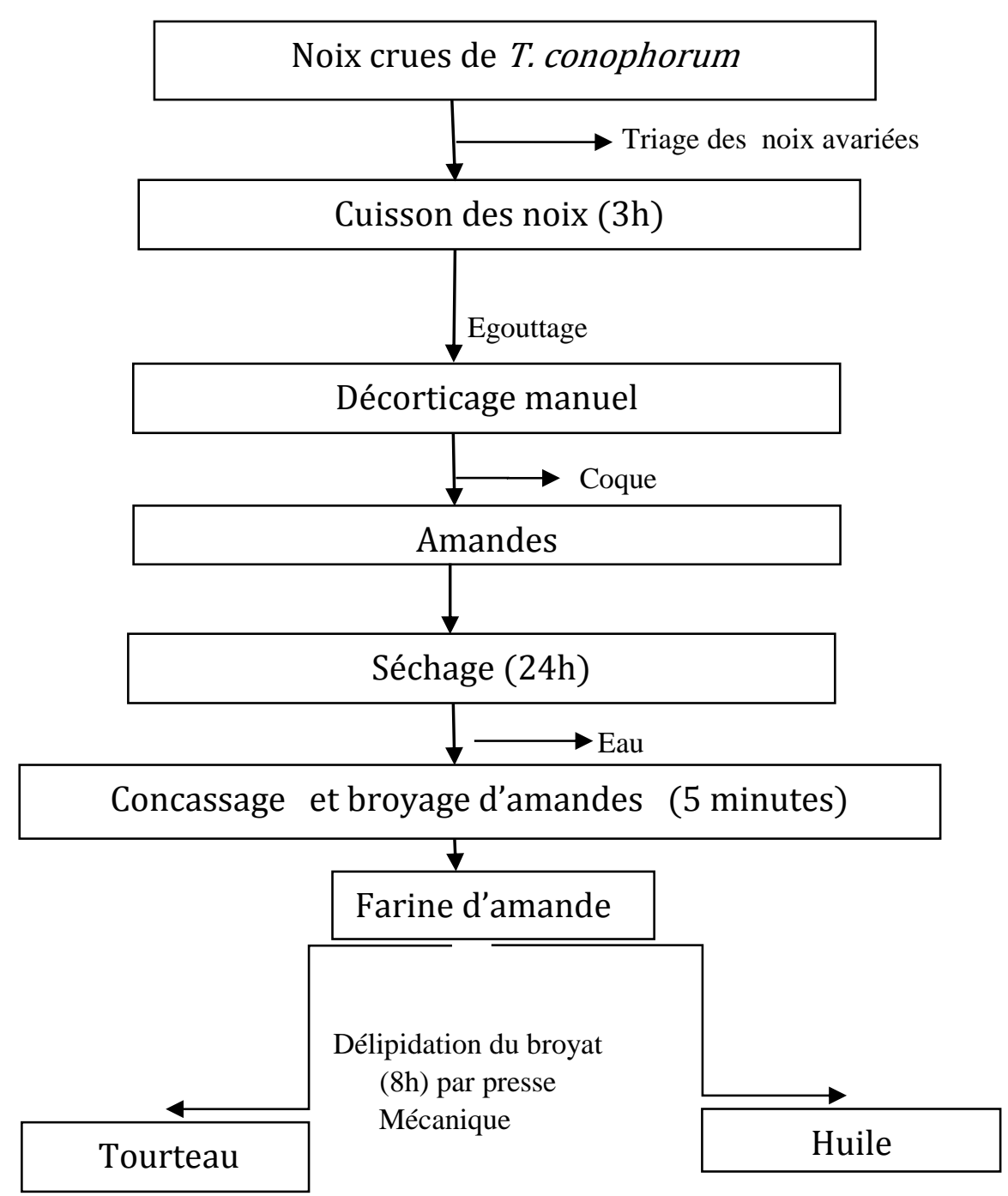

Figure 2 : Processus de production du tourteau de T. conophorum. 
Tableau 3: Variation de la température et de l'hygrométrie observée.

\begin{tabular}{lcccc}
\hline \multirow{2}{*}{ Semaine } & \multicolumn{2}{c}{ Température $\left({ }^{\circ} \mathbf{C}\right)$} & \multicolumn{2}{c}{ Hygrométrie (\%) } \\
\cline { 2 - 5 } & Matin & Soir & Matin & Soir \\
\hline 1 & $26,5 \pm 1,35$ & $28,72 \pm 0,95$ & $70,64 \pm 2,80$ & $60,20 \pm 3,25$ \\
2 & $23,20 \pm 2.01$ & $29,42 \pm 1,35$ & $69,40 \pm 6,52$ & $59,23 \pm 5,14$ \\
3 & $25,82 \pm 0,89$ & $23,2 \pm 0,86$ & $65,14 \pm 4,52$ & $54,42 \pm 5,41$ \\
4 & $28,40 \pm 2,40$ & $30,32 \pm 1,72$, & $69 \pm 6,35$ & $53,25 \pm 2,25$ \\
Moyenne et Ecart type & $25,98 \pm 1,08$ & $27,91 \pm 1,55$ & $68,01 \pm 5,54$ & $56,78 \pm 3,40$ \\
\hline
\end{tabular}

\section{RESULTATS}

\section{La température et humidité enregistrées}

Les moyennes des températures et d'humidité enregistrées durant la période d'étude sont consignées dans le Tableau 3. Quelques variations ont été observées tant sur la température que sur l'hygrométrie à des moments alternatifs du jour.

Effet de la substitution du tourteau de soja par le tourteau de Tetracarpidium conophorum sur la mortalité

Aucun cas de mortalité n'a été enregistré dans tous les trois (3) lots expérimentaux. Le cadre expérimental a été garanti par une bonne conduite mettant les poules à l'abri des perturbations physiologiques.

Effet de la substitution du tourteau de soja par le tourteau de Tetracarpidium conophorum sur la consommation d'aliment, d'eau et l'indice de consommation

Les résultats relatifs à la consommation d'aliment, d'eau et l'indice de consommation sont consignés dans le Tableau 4.

Effet de la substitution du tourteau de soja par le tourteau de Tetracarpidium conophorum sur la consommation volontaire d'aliment

Dans l'ensemble, l'incorporation du Tourteau Tetracarpidium conophorum à $12 \%$ dans les rations a amélioré significativement la consommation volontaire d'aliment $(\mathrm{P}<0,05)$. Il a été constaté une légère augmentation de la consommation moyenne d'aliment par sujet chez les traités du lot $\mathrm{n}^{\circ} 2$ soit $+4,31 \%$ par contre elle est restée inférieure chez les traités $\mathrm{du}$ lot $\mathrm{n}^{\circ} 1$ soit 1,29\%. En revanche, l'incorporation du T. conophorum a induit une augmentation de la consommation des aliments de $+18,1 \%$ et $+8,34 \%$ à la première semaine respectivement chez les traités 2 et 1 . Cette augmentation a été observée jusqu'à la $3^{\text {ème }}$ semaine puis à la $4^{\mathrm{ème}}$ semaine, elle a baissé chez les traités 2 .

Effet de la substitution du tourteau de soja par le tourteau de Tetracarpidium conophorum sur la consommation volontaire d'eau

La consommation d'eau a été meilleure aussi bien chez les témoins que chez les traités (T12). Tout comme la consommation volontaire d'eau, les effets significatifs $(\mathrm{P}<0,05)$ ont été observés d'un traitement à l'autre.

Effet de la substitution du tourteau de soja par le tourteau de Tetracarpidium conophorum sur l'indice de consommation

Selon les observations du Tableau 4, il ressort d'une manière générale que l'incorporation partielle ou totale du tourteau de T. conophorum a amélioré significativement l'indice de consommation. 
Effet de la substitution du tourteau de soja par le tourteau de Tetracarpidium conophorum sur les variables de ponte

Les résultats obtenus notamment le nombre d'œufs, le poids de l'œuf, la masse d'œufs et le taux de ponte sont exprimés en moyenne par sujet et présentés dans le Tableau 5.

\section{Effet de la substitution du tourteau de soja par} le tourteau de Tetracarpidium conophorum sur la production des oufs

D'une manière globale, la production des œufs a évolué avec l'âge des sujets.

Elle a été cependant variable d'une semaine à l'autre chez les traités1. Les données moyennes ont montré que la substitution totale ou partielle a engendré une augmentation du nombre d'œufs par sujet par semaine de $+5,86 \%$ (T2) et $+1,62 \%(\mathrm{~T} 1)$. De la première à la quatrième semaine, le lot 2 a produit plus d'œufs par semaine.

Effet de la substitution du tourteau de soja par le tourteau de Tetracarpidium conophorum sur le taux de ponte

Durant toute l'expérimentation, ce taux a été largement supérieur chez les T2 suivi des $\mathrm{T} 1$ avec respectivement $+10,12 \%$ et $+2,55 \%$ par rapport aux témoins.

Effet de la substitution du tourteau de soja par le tourteau de Tetracarpidium conophorum sur le poids des oeufs

Globalement, il ressort que, le poids de l'œuf est inférieur chez les traités avec des différences non significatives par rapport aux témoins chez lesquels il s'est amélioré avec l'âge. De la première à la $4^{\text {ème }}$ semaine de ponte, le poids moyen de l'œuf a connu une variation à l'intérieur de chaque groupe et entre les groupes expérimentaux.

Effet de la substitution du tourteau de soja par le tourteau de Tetracarpidium conophorum sur la masse d'oufs

Les résultats du Tableau 5 soulignent que le tourteau de $T$. conophorum incorporé à
$12 \%$ dans la ration des poules pondeuses a amélioré la masse d'œufs en rapport avec le nombre d'œufs pondus. Ce qui confirme l'efficacité alimentaire de la substitution totale du tourteau de soja par du tourteau de $T$. conophorum.

Effet de la substitution du tourteau de soja par le tourteau de Tetracarpidium conophorum sur les variables économiques

Les coûts de production de l'aliment, de l'œuf et le prix de revient de l'œuf sont consignés dans les Tableaux 6 et 7.

Effet de la substitution du tourteau de soja par le tourteau de Tetracarpidium conophorum sur le coût de production de l'aliment

Du Tableau 6, il apparait que le coût de production d'aliment a évolué avec l'incorporation du tourteau T. conophorum. Plus le taux de substitution est élevé, plus l'on assiste à une réduction du coût de production d'aliment, soit -15,1 FCFA pour les traités 1 et $-37,2$ FCFA pour les traités 2, par kg d'aliment. Cette amélioration est principalement due à l'utilisation du T. conophorum qui coûte moins cher que le tourteau soja.

Effet de la substitution du tourteau de soja par le tourteau de Tetracarpidium conophorum sur le coût de production de l'ouf

Le coût de production et le prix de vente de l'œuf ont été ensuite estimés et présentés au Tableau 7. Les résultats relatifs à l'estimation du coût de production laissent constater que la substitution partielle et totale du tourteau soja a diminué le coût de production de l'œuf de $-7,19 \%$ et $-7,07 \%$ respectivement chez les $\mathrm{T} 1$ et $\mathrm{T} 2$.

Effet de la substitution du tourteau de soja par le tourteau de T. Conophorum sur le prix de revient de l'ouf

Tout comme les coûts de production de l'aliment et de l'œuf, le prix de revient d'un œuf a diminué de $-11,20 \%$ pour les traités 2 et de- 4 , $54 \%$ pour les traités 1 . 
Tableau 4 : Effet de la substitution du tourteau de soja par le tourteau de T. conophorum sur la consommation d'aliment, d'eau l'indice de consommation et l'indice de conversion.

\begin{tabular}{|c|c|c|c|c|}
\hline \multirow[b]{2}{*}{ Semaine } & \multirow[b]{2}{*}{ Age (jour) } & \multicolumn{3}{|c|}{ Lots } \\
\hline & & T0 & T1 & $\mathbf{T 2}$ \\
\hline \multicolumn{5}{|c|}{ Consommation volontaire d'aliment (g/j/poule) } \\
\hline 1 & 249 & $90,20 \pm 15,47^{c}$ & $106,63 \pm 23,01^{b}$ & $134,49 \pm 54,97^{\mathrm{a}}$ \\
\hline 2 & 256 & $107,86 \pm 9,72^{\mathrm{b}}$ & $100,47 \pm 7,46^{\mathrm{b}}$ & $118,98 \pm 14,03^{\mathrm{a}}$ \\
\hline 3 & 263 & $105,96 \pm 7,62^{b}$ & $97,82 \pm 12,33^{b}$ & $103,88 \pm 10,04^{\mathrm{a}}$ \\
\hline 4 & 270 & $112,12 \pm 3,61^{\mathrm{c}}$ & $100,65 \pm 4,53^{b}$ & $96,33 \pm 9,69^{\mathrm{a}}$ \\
\hline \multicolumn{2}{|c|}{ Moyenne } & $104,04 \pm 9,57^{\mathrm{b}}$ & $101,39 \pm 3,73^{\mathrm{b}}$ & $113,42 \pm 16,91^{\mathrm{a}}$ \\
\hline \multicolumn{5}{|c|}{ Consommation volontaire d'eau (ml/j/poule) } \\
\hline 1 & 249 & $221,63 \pm 74,94^{c}$ & $271,94 \pm 118,66^{\mathrm{b}}$ & $337,06 \pm 113,92^{a}$ \\
\hline 2 & 256 & $386,73 \pm 47,99^{\mathrm{c}}$ & $362,24 \pm 40,77^{b}$ & $311,84 \pm 157,54^{\mathrm{a}}$ \\
\hline 3 & 263 & $363,27 \pm 60,11^{\mathrm{c}}$ & $351,02 \pm 64,87^{b}$ & $391,43 \pm 68,62^{\mathrm{a}}$ \\
\hline 4 & 270 & $413,27 \pm 19,53^{\mathrm{a}}$ & $302,04 \pm 65,17^{b}$ & $425,92 \pm 44,93^{\mathrm{a}}$ \\
\hline \multicolumn{2}{|c|}{ Moyenne } & $346,22 \pm 85,54^{\mathrm{c}}$ & $321,81 \pm 42,29^{b}$ & $366,56 \pm 51,66^{\mathrm{a}}$ \\
\hline \multicolumn{5}{|c|}{ Indice de consommation par poule } \\
\hline 1 & 249 & $2,54^{\mathrm{b}}$ & $2,91^{\mathrm{a}}$ & $2,87^{\mathrm{a}}$ \\
\hline 2 & 256 & $2,95^{\mathrm{a}}$ & $2,33^{\mathrm{b}}$ & $2,77^{\mathrm{a}}$ \\
\hline 3 & 263 & $2,82^{\mathrm{b}}$ & $2,12^{\mathrm{a}}$ & $2,24^{\mathrm{a}}$ \\
\hline 4 & 270 & $2,91^{\mathrm{b}}$ & $1,87^{\mathrm{a}}$ & $2,08^{\mathrm{a}}$ \\
\hline \multicolumn{2}{|c|}{ Moyenne } & $2,80 \pm 0,19^{c}$ & $2,31 \pm 0,45^{\mathrm{b}}$ & $2,49 \pm 0,39^{\mathrm{a}}$ \\
\hline
\end{tabular}

Sur une même ligne, deux moyennes portant deux lettres différentes en exposant sont statistiquement différentes $(\mathrm{P}<0.05)$.

Tableau 5: Effet de la substitution du tourteau de soja par du tourteau de T. conophorum sur les variables de ponte.

\begin{tabular}{lcccc}
\hline \multirow{2}{*}{ Semaine } & \multicolumn{4}{c}{ Production hebdomadaire par poule en moyenne } \\
\cline { 2 - 5 } & Age en jour & T0 & T1 & T2 \\
\hline 1 & 249 & $4,57 \pm 0,98^{\mathrm{a}}$ & $5,86 \pm 0,90^{\mathrm{b}}$ & $4,86 \pm 2,97^{\mathrm{a}}$ \\
2 & 256 & $5,29 \pm 0,95^{\mathrm{b}}$ & $5,14 \pm 1,95^{\mathrm{b}}$ & $6,00 \pm 0,82^{\mathrm{a}}$ \\
3 & 263 & $5,57 \pm 0,53^{\mathrm{b}}$ & $5,71 \pm 0,49^{\mathrm{b}}$ & $6,86 \pm 0,38^{\mathrm{a}}$ \\
4 & 270 & $6,43 \pm 0,53^{\mathrm{a}}$ & $5,86 \pm 0,90^{\mathrm{b}}$ & $6,86 \pm 0,38^{\mathrm{a}}$ \\
Moyenne & & $5,46 \pm 0,77^{\mathrm{b}}$ & $5,64 \pm 0,34^{\mathrm{b}}$ & $6,14 \pm 0,95^{\mathrm{a}}$ \\
\hline
\end{tabular}

\begin{tabular}{|c|c|c|c|c|}
\hline \multicolumn{5}{|c|}{ Poids moyens des œufs pondus (g) } \\
\hline 1 & 249 & $56,07 \pm 2,47^{\mathrm{a}}$ & $55,94 \pm 2,64^{\mathrm{a}}$ & $55,00 \pm 2,21^{a}$ \\
\hline 2 & 256 & $57,08 \pm 1,51^{\mathrm{a}}$ & $58,32 \pm 1,49^{a}$ & $57,16 \pm 1,65^{\mathrm{a}}$ \\
\hline 3 & 263 & $57,92 \pm 1,97^{\mathrm{a}}$ & $56,88 \pm 1,19^{b}$ & $58,76 \pm 1,54^{a}$ \\
\hline
\end{tabular}




\begin{tabular}{lcccc}
4 & 270 & $58,65 \pm 0,96^{\mathrm{b}}$ & $55,42 \pm 0,86^{\mathrm{a}}$ & $56,53 \pm 1,07^{\mathrm{a}}$ \\
Moyenne & \multicolumn{5}{c}{$57,43 \pm 1,11^{\mathrm{a}}$} & $56,64 \pm 1,28^{\mathrm{a}}$ & $56,86 \pm 1,55^{\mathrm{a}}$ \\
\hline \multicolumn{4}{c}{ Masse moyenne des œufs pondus par poule $(\mathrm{g})$} \\
\hline 2 & 249 & $256,25 \pm 48,19^{\mathrm{a}}$ & $327,80 \pm 58,66^{\mathrm{b}}$ & $267,32 \pm 81,33^{\mathrm{a}}$ \\
3 & 256 & $301,93 \pm 52,50^{\mathrm{b}}$ & $299,79 \pm 37,01^{\mathrm{b}}$ & $342,96 \pm 137,60^{\mathrm{a}}$ \\
4 & 263 & $322,61 \pm 52,50^{\mathrm{b}}$ & $324,80 \pm 38,09^{\mathrm{b}}$ & $403,08 \pm 153,36^{\mathrm{a}}$ \\
Moyenne & 270 & $377,11 \pm 24,88^{\mathrm{a}}$ & $324,75 \pm 55,03^{\mathrm{b}}$ & $387,78 \pm 145,29^{\mathrm{a}}$ \\
\hline \multicolumn{5}{c}{ Taux moyen de ponte $(\%)$} \\
\hline 1 & $65,31 \pm 11,24^{\mathrm{b}}$ & $83,67 \pm 12,85^{\mathrm{a}}$ & $83,33 \pm 16,70^{\mathrm{a}}$ \\
2 & 249 & $73,47 \pm 12,85^{\mathrm{b}}$ & $79,59 \pm 7,64^{\mathrm{b}}$ & $85,71 \pm 12,78^{\mathrm{a}}$ \\
3 & 256 & $83,67 \pm 17,36^{\mathrm{b}}$ & $87,76 \pm 9,86^{\mathrm{b}}$ & $97,96 \pm 5,40^{\mathrm{a}}$ \\
4 & 263 & $97,96 \pm 5,40^{\mathrm{a}}$ & $79,59 \pm 13,94^{\mathrm{b}}$ & $93,88 \pm 7,64^{\mathrm{a}}$ \\
Moyenne & 270 & $80,10 \pm 14,08^{\mathrm{b}}$ & $82,65 \pm 3,91^{\mathrm{b}}$ & $90,22 \pm 6,86^{\mathrm{a}}$ \\
\hline \multicolumn{4}{c}{ Sur une même ligne, deux moyennes portant deux lettres différentes en exposant sont statistiquement différentes $(\mathrm{P}<0.05)}$.
\end{tabular}

Tableau 6: Coût de revient de l'aliment ponte.

\begin{tabular}{|c|c|c|c|c|}
\hline \multirow{2}{*}{ Composition d'ingrédients } & \multirow{2}{*}{$\begin{array}{c}\text { Prix unitaire } \\
\text { (FCFA /kg) }\end{array}$} & \multicolumn{3}{|c|}{ Prix Traitement (FCFA) } \\
\hline & & Témoin & Traité1 & Traité 2 \\
\hline Maïs grain jaune & 270 & 5400 & 5400 & 5400 \\
\hline Farine de graines de mil & 150 & 1650 & 1500 & 1650 \\
\hline Farine grossière de manioc & 100 & 1400 & 1300 & 1400 \\
\hline Son de blé & 300 & 3450 & 3450 & 3450 \\
\hline Huile de palme & 700 & 1400 & 1400 & 1400 \\
\hline Farine de poisson & 400 & 4000 & 4000 & 4000 \\
\hline Farine de graines de Niébé & 300 & 3600 & 4200 & 3600 \\
\hline Tourteau de $T$. conophorum & 300 & 0 & 1800 & 3600 \\
\hline Tourteau de soja & 610 & 7320 & 3660 & 0 \\
\hline Calcaire & 120 & 840 & 840 & 840 \\
\hline Sel de cuisine & 400 & 160 & 160 & 160 \\
\hline Complexe minéral, aminé vitaminé & 15000 & 1500 & 1500 & 1500 \\
\hline Transport et manutention & & 2500 & 2500 & 2500 \\
\hline Coût de $100 \mathrm{~kg}$ d'aliment & & 33220 & 31710 & 29500 \\
\hline Coût de production d'un $\mathrm{kg}$ d'alime & FA) & 332,2 & 317,1 & 295 \\
\hline
\end{tabular}


Tableau 7: Coût de production et prix de vente de l'œuf.

\begin{tabular}{lcccc}
\hline \multirow{2}{*}{ Désignation } & \multicolumn{3}{c}{ Coût de traitement } \\
\cline { 3 - 5 } & & Témoin & Traité 1 & Traité 2 \\
\hline Charge d'aliment de ponte & 53,51 & 41,50 & 41,69 \\
& Soins vétérinaires & 0,8 & 0,8 & 0,8 \\
\multirow{2}{*}{ Autres charges } & Main d'œuvre permanente & 4,66 & 4,66 & 4,66 \\
& Conditionnement & 1,55 & 1,55 & 1,55 \\
& Transport & 2,72 & 2,72 & 2,72 \\
\multirow{2}{*}{ Coût global de production (CP) de l'œuf } & 3,45 & 3,45 & 3,45 \\
Prix de vente de l'œuf & Charges et pertes diverses & 18,81 & 18,81 & 18,81 \\
\hline
\end{tabular}

*Bâtiment, matériel et poules-pondeuses.

\section{DISCUSSION}

Aucune donnée n'est disponible dans la littérature sur l'utilisation du tourteau de $T$. conophorum dans l'alimentation des poules pondeuses d'où, l'originalité de cette étude. C'est pour cette raison que la présente discussion portera uniquement sur la comparaison avec d'autres tourteaux comme les tourteaux de courge, sésame, de safou et de coton, toutefois moins riches en MAT que le $T$. conophorum. Il sied aussi de noter que dans les conditions expérimentales de cette étude, la composition chimique de $T$. conophorum a été de 33,95\% MAT (Ossoko et al., 2021). Ce taux est proche de celui obtenu dans les précédentes études soit 52\% MAT (Mezajoug kenfack, 2010) et plus élevé que celui des autres tourteaux locaux non conventionnels (Adzona, 2019).

\section{La température et l'humidité enregistrées}

La température moyenne de $27,57^{\circ} \mathrm{c}$ observée par jour a été largement supérieure à celle de la zone thermique de tolérance comprise entre 18 et $22^{\circ} \mathrm{c}$ (Techniques et sciences avicoles, 2004). Ces résultats corroborent ceux obtenus par Brou et al. (2012), soit une température moyenne de $27,92^{\circ} \mathrm{c}$ à la fin d'étude évaluant l'effet de la variation temporelle de la température ambiante journalière sur le poids des œufs de poules pondeuses ISA Brown en Côte-d'Ivoire. Par contre, ces données sont en deçà de celles prélevées par Mantsanga et al. (2016) dont les moyennes de températures étaient de $28,98^{\circ} \mathrm{C}$ le matin et $30,04^{\circ} \mathrm{C}$ le soir durant toute la période d'expérimentation en alimentation calcique séparée chez les poules pondeuses ISA Brown au Congo.

Les variations de la température ambiante observées durant l'expérimentation n'ont pas influencé directement sur les variables de ponte. Cela signifie : malgré que les poules soient les animaux homéothermes accusant la température interne assez élevée et comprise entre 40 et $42^{\circ} \mathrm{C}$, cette température reste cependant constante quelle que soit la température extérieure. Leur métabolisme élevé et leur plumage participent à cette régulation surtout quand les conditions ambiantes optimales leur sont offertes (Brou et al., 2012).

En revanche, la moyenne d'humidité relative de $62,39 \%$ constatée par jour a été en deçà de l'hygrométrie requise située entre 68$70 \%$ (FAO, 2004). Ce taux ne peut influencer sur la production qu'en rapport avec l'augmentation de la température impactant ainsi l'humidité spécifique (Sciences et Techiques Avicoles, 2004).

Effet de la substitution du tourteau de soja par le tourteau de Tetracarpidium conophorum sur la mortalité

La mortalité nulle observée chez les traités montre que l'incorporation du tourteau 
de T. conophorum jusqu'à $12 \%$ dans l'alimentation des poules pondeuses n'a eu aucun effet toxique sur les sujets.

Ces résultats sont en accord avec ceux obtenus par Houndonougbo et al. (2012) qui, en évaluant les performances bioéconomiques des poulettes alimentées avec des rations à base de feuilles séchées de manioc (Manihot esculenta) n'ont enregistré aucune mortalité chez les poulettes.

Effet de la substitution du tourteau de soja par le tourteau de Tetracarpidium conophorum sur la consommation volontaire d'aliment

Les résultats obtenus sur la consommation volontaire d'aliment sont supérieurs à ceux obtenus par Saboukoulou (2020) soit 91,63 g/jour/poule et ceux de Guédou et al. (2012), soit 61,6 g/jour/poule en valorisant le tourteau palmiste, coton et les graines de coton dans une ration des poules pondeuses.

La consommation volontaire moyenne obtenue est légèrement éloignée des travaux antérieurs (Mantsanga et al., 2016). Ceci peut être assimilé aux conditions climatiques comme la température qui, au cours de cette expérimentation étaient toujours élevées. Par ailleurs, la légère diminution de la consommation d'aliment observée chez les traités (T12) à la quatrième semaine stipule que malgré le traitement thermique appliqué, certains facteurs antinutritionnels présents dans le $T$. conophorum auraient échappé à la cuisson et à la torréfaction.

Effet de la substitution du tourteau de soja par le tourteau de Tetracarpidium conophorum sur la consommation volontaire d'eau

L'augmentation de la consommation d'eau induite, du fait de l'incorporation du tourteau $T$. conophorum $(\mathrm{P}<0,05)$ explique la forte consommation d'aliment. Les résultats des deux lots expérimentaux sont largement supérieurs à ceux obtenus par Saboukoulou (2020) soit 188,58 ml/jour/poule et à ceux de Mantsanga et al. (2016) soit $283 \mathrm{ml} /$ jour/poule chez les poules pondeuses Lohman nourries avec un aliment calcique séparé. Ces différences d'augmentation signifient tout simplement que la consommation d'eau va de pair avec la consommation d'aliment. D'ailleurs, pour une poule en ponte la consommation d'eau est estimée à $300 \mathrm{ml}$ par jour (Mantsanga et al., 2016).

Effet de la substitution du tourteau de soja par le tourteau de Tetracarpidium conophorum sur l'indice de consommation.

L'amélioration significative de l'indice de consommation constatée explique que l'utilisation du tourteau de $T$. conophorum a amélioré la digestibilité d'aliment. Ces indices obtenus sont meilleurs à ceux obtenus par Saboukoulou (2020), soit 3,11 et Guédou et al (2018) soit 3,20 qui ont valorisé respectivement le tourteau de la pulpe de Dacryodes edulis. Ces indices prouvent à suffisance l'efficacité alimentaire du tourteau de T. conophorum.

\section{Effet de la substitution du tourteau de soja par le tourteau de Tetracarpidium conophorum sur la production des oufs}

La production des œufs a évolué avec l'âge des sujets tant chez les traités 2 que chez les témoins ; elle a été cependant variable d'une semaine à l'autre chez les traités 1 .

De la première à la quatrième semaine, le lot 2 a produit plus d'œufs par semaine. Par contre le nombre d'œufs produits par sujet du lot 1 est inférieur. D'une manière globale, les données moyennes ont montré que la substitution a engendré une augmentation du nombre d'œufs par sujet par semaine de $+5,86 \%$ (T2) et $+1,62 \%$ (T1). Cela suppose quelques facteurs antinutritionnels persistant dans le tourteau de T. conophorum n'ont pas influencé négativement sur la productivité des poules. Ainsi donc, la teneur en facteurs antinutritionnels des aliments expérimentaux serait dans le seuil de tolérance des poules pondeuses comme l'affirment Brah et al. (2019).

Ces résultats concordent à ceux obtenus par Mantsanga et al. (2016), en alimentation calcique séparée, soit une moyenne de 5 œufs par poule par semaine. 
Effet de la substitution du tourteau de soja par le tourteau de Tetracarpidium conophorum sur le poids des œufs

Les différences de poids moyen de l'œuf entre ces deux traitements observées n'ont pas été statistiquement significatives, soit : $57,43 \pm 1,11 \mathrm{~g}$ (T0), $56,64 \pm 1,28 \mathrm{~g}$ (T1) et $56,86 \pm 1,55 \mathrm{~g}$ (T12). Ces résultats sur le poids corroborent à ceux de Hien et al. (2017) qui en analysant les effets d'une ration à base de la variété de maïs «Espoir » sur la productivité des poulettes, les poids étaient centrées dans l'intervalle $49,0 \pm 2,9 \mathrm{~g}$.

Les résultats des différents traitements sont en accord avec ceux réalisés par Saboukoulou (2020). Ce dernier auteur en substituant partiellement le tourteau de soja par du tourteau de safou chez la même souche a obtenu des différences non significatives entre les témoins $(56,41 \mathrm{~g} \pm 1,28)$ et (traités $56,48 \mathrm{~g}$ $\pm 1,58$ ).

Ces résultats sont différents et supérieurs de ceux trouvés par Matsanga et al. (2016) chez qui, l'effet de l'alimentation calcique séparée sur les performances de production de la poule Lhoman Brown sous le climat tropical humide a produit de réponses entre traitements significativement déférentes, soit $52,83 \pm 4,48 \mathrm{~g}$ (témoin) et 54,67 $\pm 5,45 \mathrm{~g}$ (traité)

Cela explique l'efficacité alimentaire de l'incorporation du tourteau $T$. conophorum qui améliore la digestibilité.

\section{Effet de la substitution du tourteau de soja} par le tourteau de Tetracarpidium conophorum sur la masse d'oufs

Les résultats soulignent que le tourteau de $T$. conophorum incorporé à $12 \%$ dans la ration des poules pondeuses a amélioré la masse d'œufs en rapport avec le nombre d'œufs pondus. Ce qui confirme bien l'efficacité alimentaire de la substitution totale du tourteau de soja par du tourteau de T. conophorum.

\section{Effet de la substitution du tourteau de soja par le tourteau de Tetracarpidium conophorum sur le taux de ponte}

Durant toute l'expérimentation, ce taux a été largement supérieur chez les T2 suivi des
T1 avec respectivement $+10,12 \%$ et $+2,55 \%$ par rapport aux témoins. Le taux de ponte a été également élevé aussi pour les traités que les témoins des expériences antérieures réalisées par Saboukoulou (2020), soit 74,3\% par Guédou et al. (2018) soit 75,74\%.

Ce même taux est proche de celui obtenu par Dongmo et al. (2012) soit 80,8\% qui ont incorporé le tourteau de coton dans l'aliment des poules pondeuses.

Ces résultats obtenus dans cette étude se justifient par la souche utilisée, l'âge des poules, le milieu d'étude (station) et surtout la qualité de l'aliment qui a influencé sur la production des œufs.

Ce même taux est proche de celui obtenu par Dongmo et al. (2012), soit 80,8\% qui ont incorporé le tourteau de coton dans l'aliment des poules pondeuses.

Ces résultats obtenus dans cette étude se justifient par la souche utilisée, l'âge des poules, le milieu d'étude (station) et surtout la qualité de l'aliment qui a influencé sur la production des œufs.

\section{Effet de la substitution du tourteau de soja par le tourteau de Tetracarpidium conophorum sur le coût de production de l'aliment}

Le résultat de la substitution totale enregistré s'accorde avec celui obtenu par Saboukoulou (2020) qui, en substituant le tourteau de soja par le tourteau de safou dans l'alimentation a obtenu 276 FCFA pour $1 \mathrm{~kg}$ d'aliment. Dans l'ensemble, les aliments formulés ont diminué le prix de revient d'un $\mathrm{kg}$ d'aliment aussi bien chez les témoins que chez les traités par rapport au prix du $\mathrm{kg}$ de concentré sur le marché qui s'élève à 400 FCFA (Mantsanga et al., 2016).

Ces résultats sont meilleurs que ceux obtenus par Mars et Peter (2021) qui, en substituant le tourteau de soja par les tourteaux colza et tournesol, de maïs, du pois protéagineux et de la protéine de pomme de terre soumis à de fortes fluctuations de prix a obtenu un surcoût de 3 FCFA.

Le résultat du prix de l'aliment substitué est proche à celui obtenu par Guédou et al. (2018) au Benin dont le prix de revient de 
l'aliment ponte est de 250 FCFA et par Mantsanga et al. (2016) au Congo-Brazzaville, soit 300 FCFA. Ceci peut être expliqué par l'utilisation des ingrédients locaux qui coûtent moins chers pour la plupart.

Effet de la substitution du tourteau de soja par le tourteau de Tetracarpidium conophorum sur le coût de production de l'œuf

Le résultat sur le coût de production de l'œuf est meilleur à celui obtenu par la FAO (2009b) à l'issue d'une étude sur la cartographie de la filière avicole au Congo Brazzaville, pour qui le coût de production d'un œuf de table au Congo Brazzaville s'élève à 91,32 FCFA (FAO, 2009a). Cela signifie que malgré l'incorporation importante des ingrédients non conventionnels notamment le tourteau T. conophorum, l'estimation des apports nutritifs des différentes formulations utilisées pour la présente expérience ont été en harmonie avec les normes fixées par certains tableurs aliment-volaille telle que l'affirment Brah et al. (2019). En utilisant les tableurs TOAFA avec les mêmes ingrédients alimentaires et les mêmes prix, on obtient un aliment poule pondeuse (18 semaines d'âge à plus) semblable assez équilibré en énergie (2900 kcal/kg), en protéine brute $(18 \%)$, en méthionine, en calcium, en phosphore disponible avec un taux de cellulose brute inférieur à $5 \%$.

Effet de la substitution du tourteau de soja par le tourteau de $T$. conophorum sur le prix de revient de l'œuf

La marge bénéficiaire obtenue et qui s'élève à 22,87 FCFA (T2), 19, 37 FCFA (T1) et 22,38 FCFA (T0) est meilleure à celle du marché local où le prix de vente de l'œuf en détail est à 125 FCFA avec une marge bénéficiaire estimée à 33,68 FCFA par œuf de table (FAO, 2009b).

Ces résultats montrent que l'œuf produit avec incorporation du tourteau T. conophorum procure plus de bénéfice. Cette étude montre que la substitution du tourteau de soja par du tourteau de $T$. conophorum favorise la production des œufs à moindre coût.
Ce faible coût de production suppose que la masse d'œufs a été importante chez les poules traitées pour une consommation d'aliment réduite. D'après ces résultats, le plateau de 30 œufs en alimentation à substitution totale du tourteau de soja par du tourteau de $T$. conophorum peut être vendu au Congo à un coût relativement identique à celui de la RCA et RCA (2500-2750FCFA), toujours supérieur au prix du de Mali (1500 et 1800 FCFA) et largement supérieur à celui du Sénégal (1000 et 1500FCFA) et du Cameroun (1200 et 1500 FCFA) (OFIVAL, 2005).

\section{Conclusion}

L'objectif de la présente étude était d'évaluer l'effet de la substitution du tourteau soja par du tourteau $T$. conophorum sur les performances des poules pondeuses en station. Les résultats obtenus ont souligné que le tourteau de $T$. conophorum incorporé à $12 \%$ dans la ration des poules pondeuses augmente la production d'œufs, le poids de l'œuf, la masse d'œufs et le taux de ponte et le coût de l'aliment et de l'œuf. Ce qui stipule que ce tourteau est économiquement compétitif en comparaison du tourteau de soja.

Par ailleurs, les caractéristiques des poules n'ont pas été affectées. Il a été conclu dans les conditions de la présente étude, que jusqu'à $12 \%$ de substitution, le tourteau de soja peut être remplacé par du tourteau $T$. conophorum sans impacter négativement sur les variables de la ponte et sur le plan économique. Cette substitution s'explique par le fait que les deux tourteaux ont la composition chimique similaire notamment en protéines brutes. L'hypothèse formulée au début de ce travail a été vérifiée et l'objectif atteint. L'incorporation de tourteau $T$. conophorum dans l'aliment ponte ouvre des perspectives intéressantes en aviculture fermière.

\section{CONFLIT D'INTERETS}

Les auteurs déclarent l'existence d'aucun conflit d'intérêts.

\section{CONTRIBUTIONS DES AUTEURS}

Ce travail est le fruit des efforts de l'équipe de chercheurs ayant contribué de 
plusieurs manières à la réalisation de l'expérimentation jusqu'à l'élaboration du document. VMN a effectué les descentes sur le terrain pour la commande et l'achat des noix jusqu'à leur transport sur Brazzaville, où a eu lieu l'expérimentation. IL a ensuite participé activement à la réalisation de l'expérimentation et l'élaboration de cette version d'article. TK a participé effective au suivi de l'expérience. BBMM a récolté les données. PPA: ses apports financiers, techniques et moraux durant toute cette étude ont contribué à la production de cet article. JBB: il a participé activement au traitement des données et l'élaboration du document. AFN : son apport technique a permis la fabrication des aliments expérimentaux. JRG : sa contribution la plus marquante a été la fourniture sans rupture de certains ingrédients nécessaires pour la fabrication des aliments. AJS : son apport moral et sa participation à l'amendement de ce document ont été les plus remarqués durant toute cette étude. HBM : pour son assistance permanente, son encadrement multidimensionnel et son implication déterminée à la réalisation de ce travail.

\section{REMERCIEMENTS}

Les auteurs expriment leur gratitude envers l'Institut national de Recherche Agronomique pour la fourniture du matériel animal. Ce travail a bénéficié également l'appui technique du Docteur Florence Dorothée SIANARD, Directrice de l'Agence Nationale sur Valorisation des Résultats de la Recherche (ANVAR) et Madame Diane MAVOUNGOU, Présidente de la Coopérative Santé et Nature (CSN), pour la production du tourteau.

\section{REFERENCES}

Adzona PP. 2019. Influence de quatre tourteaux locaux non conventionnels en alimentation mélangée, fractionnée et séquentielle chez la volaille en milieu tropical. Thèse de Doctorat unique, Faculté des Sciences et Techniques, Université Marien Ngouabi, Brazzaville, Congo, $133 \mathrm{P}$.

Adzona PP, Bonou GA, Bati JB, Ndinga FA, Ondjomoko LD, Itoua PL, Kiki PS, Dotchet IO, Banga-Mboko H, Abdou-
Karim I. 2019. Influence du tourteau de sésame en alimentation fractionnée séparée et séquentielle sur les performances zootechniques et économiques du poulet de chair standard de la souche Cobb 500. R.I.S.A., 2(1): 111.

Anonyme. 2019. Lékana (Ville). RDC. https://fr.wikipedia.org/w/index.ph p- title $=$ Lékana (ville) \&oldid=162542356 ». Consulté le 25 juin 2021.

Anonyme. 2021. Subdivision de la République du Congo. RDC. https://fr.wikipedia.org/wiki/R\%C3\%A9 publique_du_Congo. Consulté le 15 juin 2021.

Ayoola PB, Adeyeye A, Onawumi OO, Faboya OOP. 2011. Phytochemical and nutrient evaluation of Tetracarpidium conophorum (Nigeria Walnut) root. Int. J. Res. Rev. Appl. Sci., 7(2): 197-202.

Bernadin JR, Jiofack T, Lejol J, Tchoundjeu Z, Guedje MN. 2012. Agroforestry and socioeconomic potential of a nonconventional liana: Tetracarpidium conophorum (Müll. Arg.) Huch. \& Dalz. in Cameroon. Higher Institute of Environmental Sciences, Yaoundé, Cameroon. Tetracarpidium conophorum. Bois et Forêts des Tropiques, 313(3): 3545. DOI: https://doi.org/10.7202/1014986ar

Brah N, Houndonougbo MF, Issa S, Chrysostome CAAM. 2019. Tableur Ouest Africain de Formulation d'Aliments de Volailles (TOAFA Volaille). Int. J. Biol. Chem. Sci., 13(3): 1308-1320.

DOI: https://dx.doi.org/10.4314/ijbcs.v13i3.8

Brou Gkg, Houndonougbo FM, Aboh AB, Mensah GA. 2012. Effet de la variation temporelle de la température ambiante journalière sur le poids des œufs de poules pondeuses ISA Brown en Côte-d'Ivoire : Int. J. Biol. Chem. Sci., 6(5): 2158-2169. DOI:

http://dx.doi.org/10.4314/ijbcs.v6i5.23

Defoundoux HF, Lenga SD, Samba G. 2006. Pauvreté et Problèmes Environnementaux. Hal-01147940, 
Brazzaville, Congo, 148p.

Ezugwu NS, Anyanwu GE, Nto JN. 2021. Ameliorative Effect of The Seed of Tetracarpidium conophorum (African Walnut) on wistar Rats with Doxorubicin Induced Cardiac Toxicity. RAJ., 4(1): 349-360.

FAO. 2009a. Schéma directeur pour le développement des filières de l'élevage au Congo. FAO, Suisse, 49 p.

FAO. 2009b. Cartographie et compétitivité des filières d'élevages prioritaires au Congo. FAO, Suisse, 56p.

Guedou MSE, Kouato GO, Houndonougbo MF, Chrysostome CAAM, Mensah GA. 2018. Performances de ponte et qualité des œufs de poules pondeuses nourries avec des aliments à base de différentes variétés de grains de maïs. Int. J. Biol. Chem. Sci., 12(6): 2846-2855. DOI: https://dx.doi.org/10.4314/ijbcs.v12i6.29

Hien OC, Diarra B, Coulibaly Y. 2017. Effets d'une ration à base de la variété de maïs « Espoir » sur la productivité des poulettes. Int. J. Biol. Chem. Sci., 11(2): 806-816.

DOI: https://dx.doi.org/10.4314/ijbcs.v11i2.22

Houndonougbo MF, Chrysostome CAAM, Houndonougbo VP. 2012. Performances bioéconomiques des poulettes alimentées avec des rations à base de feuilles séchées de manioc (Manihot esculenta). Int. J. Biol. Chem. Sci., 6(2): 670-676. DOI: http://dx.doi.org/10.4314/ijbcs.v6i2.11

Guédou MSE, Tobada P, bégo G, Tossa I, Pomalegni SCB, Mensah GA. 2012. Valorisation des tourteaux de palmiste et de coton et des graines de coton dans des rations alimentaires pour des poules pondeuses au Sud-Bénin. Bulletin de la Recherche Agronomique du Bénin (BRAB), 38-44. http://www.slire.net.

ITAVI. 2015. Situation de la production et des marchés des œufs et des produits d'œufs. ITAVI, France, 8p. www.itavi.asso.fr/content/les-indicesitavi

ITAVI. 2020. Situation du marché des œufs et ovoproduits. ITAVI, France, 11p. www.itavi.asso.fr/content/les-indicesitavi
ITAVI. 2021. Indices coût matières premières dans l'aliment. ITAVI, France, 2p. www.itavi.asso.fr/content/les-indicesitavi.

Livet A, Daspres N, Lepeule C, Bordeaux C. 2015. Tourteau de sésame. Etat des lieux et perspectives de développement d'une filière pour l'alimentation avicole biologique. Avial. Bio, Alim., 2: 12.

Malanda LJ. 2016. Effet de l'incorporation du tourteau Tetracarpidium conophorum (Mull-Arg) Hutch et Dalziel, dans les aliments de démarrage et croissance des poulets de chair standard. Mémoire présenté en vue de l'obtention du diplôme de master en Sciences Agronomiques, Ecole Nationale Supérieure d'Agronomie et de Foresterie, Université Marien Ngouabi, Brazzaville, Congo, 39p.

Mantsanga HB, Amona I, Banga-Mboko H, Bakana MA, Adzona PP. 2016. Effet de l'alimentation calcique séparée sur lesperformances de production de la poule Lhoman Brown sous climat tropical humide. J. Appl. Biosci., 97: 9212-9219. DOI: http://dx.doi.org/10.4314/jab.v97i1.6

Marc R, Piter SB. 2021. Substitution du soja dans l'engraissement de volaille. HAFL. 5: 11-13.

Mezajoug KLB. 2010. Propriétés nutritionnelles et fonctionnelles des protéines de tourteaux, de concentrats et d'isolats de Ricinodendron heudelotii (Bail.) Pierre ex Pax et de Tetracarpidium conophorum (Müll. Arg). Thèse de Doctorat, Institut National Polytechnique de Lorraine, Spécialité Procédés Biotechnologiques et Alimentaires, Université de N'Gaoundéré, Cameroun, $188 \mathrm{p}$.

Ngobiri NC, Kikanme KN. 2020. Inhibition of mild steel corrosion in $0.25 \mathrm{M} \mathrm{H}_{2} \mathrm{SO}_{4}$ Using Tetracarpidium conophorum Shell Extract. JERR., 11(4): 19-24. DOI: 10.9734/JERR/2020/v11i417066

OFIVAL. 2005. Note technique sur la filière avicole. OFIVAL, Espagne, 20p.

Ojokoh E, Ojokoh LO, Nwachukwu CM, Omunu R, Eletuanya JC. 2020. Proximate Composition, Phytochemical and Mineral 
Analysis of Cooked and Raw Walnut (Tetracarpidium conophorum). Asi. Food. Sci. J., 19(2): $\quad 11-17$. DOI:10.9734/AFSJ/2020/v19i230234

Oriakhi K, Uadia PO. 2020. Hepatoprotective fractions from methanol extract of Tetracarpidium conophorum (African walnut). Arch. Bas. App. Med., 8: 45 - 48 . DOI: $10.1111 /$ jfbc. 13288

Ossoko JPL, Dzondo MG, Miakayizila BDE, Mvoula Tsieri MD. 2021. Assessment of the nutritional potential of the kernels of the seeds of Tetracarpidium conophorum collected in lékana in the department of plateaux in the Republic of Congo. J. Bio. Innov., 10(4): 1102-1110. DOI: https://doi.org/10.46344/JBINO.2021.V1 0i04.17

Roinsard A, Bordeaux C, Luba CS, Brachet MN, Germain K, Juin H. 2016. Valorisation de matières premières locales pour l'alimentation des poulets en agriculture biologique. TEMA., 4: 23-31.

Saboukoulou AJ. 2020. Effet de la Substitution partielle du tourteau de soja par le tourteau de la pulpe de Dacryodes edulis dans l'alimentation des poules pondeuses de souche Lohman Brown. Mémoire présenté en vue de l'obtention du diplôme Master Recherche en Sciences Biologiques, Option Croissance et Productions Animales, Faculté des Sciences et Techniques, Université Marien Ngouabi, Brazzaville, 71 p.

Sianard FD. 2010. Inventaire des plantes psychotropes du Congo, des recettes de désintoxication et études chimique et pharmacologique de Indigofera capitata kotschy et Tetracarpidium conophorum (Mull-Arg) Hutch. \& Dalziel. Thèse de Doctorat, Chimie - Technologie Modélisation, Faculté des Sciences et Techniques, Université Marien Ngouabi, Brazzaville, 152p.

TSA (Techniques et Sciences Avicoles). 2004. Prévention du coup de chaleur en aviculture. Rev. Sci. Avi., 68.

Uchechukwu-Nkeirukayvonne C. 2017. Phytochemical and Proximate Compositions of Tetracarpidium conophorum [African Walnut] Seeds. Int. J. Res. Stud. Biosci., 5(10): 25-31. DOI: http://dx.doi.org/10.20431/23490365.0510005 